\title{
Atividades registradas por profissionais de saúde da família no sistema de informação da atenção básica
}

Activities registered by family health professionals in the primary care information system

Actividades registradas por profesionales de salud familiar en el sistema de información de atención básica

Ricardo Bezerra Cavalcante ${ }^{1}$, Tarcísio Laerte Gontijo², Eliete Albano de Azevedo Guimarães ${ }^{3}$, Valéria Conceição de Oliveira ${ }^{4}$, Jéssica Rauane Teixeira Martins ${ }^{5}$

\footnotetext{
${ }^{1}$ Enfermeiro, Doutor em Ciência da Informação. Professor Adjunto da Universidade Federal de São João Del-Rei (UFSJ). Divinópolis, MG, Brasil. E-mail:

ricardocavalcanteufmg@yahoo.com.br.

2 Enfermeiro, Doutor em Ciências da Saúde. Professor Adjunto da UFSJ. Divinópolis, MG, Brasil. E-mail: enftarcisio@yahoo.com.br.

${ }^{3}$ Enfermeira, Doutora em Ciências da Saúde. Professora Adjunta da UFSJ. Divinópolis, MG, Brasil. E-mail: elietealbano@hotmail.com.

${ }^{4}$ Enfermeira, Doutora em Ciências da Saúde. Professora Adjunta da UFSJ. Divinópolis, MG, Brasil. E-mail: valeria.oli.enf@gmail.com.

${ }^{5}$ Discente do curso de Graduação em Enfermagem da UFSJ. Divinópolis, MG, Brasil. E-mail: jessicarauane_kzz@hotmail.com.
}

\section{RESUMO}

Estudo descritivo que analisou o registro das atividades no Sistema de Informação da Atenção Básica, realizadas por profissionais das equipes de saúde da família, em Minas Gerais. Os dados foram obtidos junto ao Departamento de Informática do Sistema Único de Saúde. Realizou-se análise descritiva das informações segundo tipo de atividade e categoria profissional, utilizando-se software estatístico SPSS ${ }^{\circledR}$ 17.0. Registraram-se 635.788 .580 atividades, sendo $81,5 \%$ de cunho individual. Atividades coletivas totalizaram $10,2 \%$ dos registros. Atendimentos em grupo e reuniões de agentes comunitários com a população totalizaram 1,1\%, e as visitas domiciliares, 7,6\%. Profissionais médicos e enfermeiros são responsáveis por $67,6 \%$ dos registros. Conclui-se que as atividades registradas são predominantemente de caráter individual, o que pode refletir a concepção do próprio sistema priorizando, na coleta, as atividades individuais, focadas em procedimentos, bem como reforçando, na disseminação das informações, esta mesma lógica.

Descritores: Sistemas de Informação; Atenção Primária à Saúde; Saúde da Família.

\section{ABSTRACT}

This descriptive study analyzed the activities registered in the Primary Care Information System, by family health professionals, in Minas Gerais. Data were obtained from the Unified Health System's IT Department. A descriptive analysis of the information was performed, by type of activity and professional category, using the statistical software SPSS $®$ 17.0. A total of $635,788,580$ activities were registered, with $81.5 \%$ of an individual nature. Collective activities totaled $10.2 \%$ of the entries. Group care and meetings of community agents with the population totaled $1.1 \%$ and home visits, $7.6 \%$ of the entries. Physicians and nurses are responsible for $67.6 \%$ of the entries. In conclusion, the activities registered are primarily individual in nature, which may reflect the system's actual design, which prioritizes collecting individual activities and those focused on procedures, and reinforces this same logic in the dissemination of information.

Descriptors: Information Systems; Primary Health Care; Family Health.

\section{RESUMEN}

Estudio descriptivo que analizó el registro de actividades realizado en el Sistema de Información de Atención Básica por profesionales de equipos de salud familiar, en Minas Gerais. Datos obtenidos del Departamento de Informática del Sistema Único de Salud. Se realizó análisis descriptivo de las informaciones, según tipo de actividad y categoría profesional, con el software SPSS $®$ 17.0. Se registraron 635.788 .580 actividades, $81,5 \%$ de carácter individual. Las actividades colectivas totalizaron el $10,2 \%$ de los registros. Las atenciones grupales y las reuniones de agentes comunitarios con la población totalizaron $1,1 \%$, y las visitas domiciliaras, 7,6\% de los registros. Los profesionales médicos y enfermeros son responsables del $67,6 \%$ de registros. Se concluye en que las actividades registradas son mayoritariamente de carácter individual, lo cual refleja la concepción del propio sistema, que prioriza la recolección de actividades individuales y enfocadas a procedimientos, así como fortalece la difusión de la información bajo similar lógica.

Descriptores: Sistemas de Información; Atención Primaria de Salud; Salud de la Familia. 


\section{INTRODUÇÃO}

Em 1994 foi implantado no Brasil o Programa de Saúde da Família que posteriormente foi denominado de Estratégia de Saúde da Família (ESF) com a finalidade de remodelação da política de atenção básica no país ${ }^{(1)}$. Buscou-se a reestruturação do modelo assistencial existente priorizando a saúde e a interdisciplinaridade, tendo como foco a família e a coletividade ${ }^{(2)}$. Essa reestruturação passa pela inversão da forma de organizar as práticas de atenção à saúde na atenção básica para atender o individuo e a família de forma integral e contínua, substituindo o modelo de práticas curativistas, por ações de promoção, proteção e recuperação da saúde de toda comunidade ${ }^{(3-4)}$.

Com a implantação desta estratégia houve necessidade de desenvolver um sistema de informação que respondesse às demandas informacionais de gestores e profissionais inseridos nesta estratégia. Nesta perspectiva, em 1998, criou-se o Sistema de Informação da Atenção Básica (SIAB) com o objetivo de instrumentalizar gestores e profissionais com informações que auxiliem no monitoramento e na avaliação das ações e dos resultados das atividades desenvolvidas pelas equipes de saúde da família ${ }^{(5)}$. Além disso, como o SIAB é utilizado para o registro de atividades desenvolvidas, permite aos gestores mensurar a produtividade das equipes, gerenciar as informações produzidas, auxiliar na construção de indicadores e fornecer subsídios para identificar a realidade e as necessidades de saúde da população(6-7).

A coleta de dados para alimentação desse sistema se dá através de visitas domiciliares e pelos atendimentos realizados na unidade de saúde e/ou domicilio ${ }^{(8)}$. A partir destes dados pode-se realizar o diagnóstico de saúde de uma determinada área de abrangência, fornecendo assim, subsídios para o planejamento e a avaliação das ações de saúde ${ }^{(6)}$.

Entretanto, a utilização do SIAB pelos profissionais apresenta dificuldades que podem ser assim relacionadas: falta de capacitação dos profissionais para trabalhar com o sistema; falta de suporte/apoio aos profissionais; o sistema possui limitações; falta de conhecimento do sistema por parte da equipe multidisciplinar ${ }^{(9)}$. Alguns estudos também têm demonstrado que as equipes de Saúde da Família não têm utilizado as informações armazenadas no SIAB no planejamento local, no processo decisório e muito menos na avaliação dos serviços ${ }^{(10-11)}$. Desta feita, o SIAB é percebido pelos profissionais como um instrumento de trabalho burocrático focado na alimentação dos níveis centrais de governo com informações de seu interesse, com pouco significado para o processo de trabalho local, das equipes de saúde da família(12-13).

Outra dificuldade relacionada ao SIAB é a sua lógica de produção de dados reconhecida pelos profissionais que 0 alimentam. Para estes profissionais os instrumentos de coleta do SIAB subsidiam um modelo de assistência focado no fluxo das consultas, nos atendimentos individuais, solicitação de exames e realização de procedimentos, o que reitera a lógica da assistência clínica, individual e curativa ${ }^{(11)}$. Isto é reforçado quando da constatação de que os instrumentos de coleta do SIAB, para o Agente Comunitário de Saúde, não são suficientes para as suas demandas de informação, pois não há como registrar nas fichas do SIAB informações relacionadas às atividades como exercícios físicos, dieta, demandas emergentes das visitas domiciliares, orientações realizadas e atividades de educação em saúde ${ }^{(14)}$. Desta forma, os ACS acabam criando outras fontes de informação como os seus "cadernos" onde registram as informações necessárias para o seu cotidiano de trabalho e que não são passíveis de registro nos instrumentos de coleta do SIAB $^{(13-14)}$.

Estas dificuldades precisam ser suplantadas com o objetivo de qualificar a informação utilizada na estratégia de saúde da família com vistas à gestão dos processos locais, bem como à produção de indicadores fidedignos.

Sabe-se que o governo federal vem propondo um aperfeiçoamento do SIAB por meio do Sistema de Informação em Saúde para a Atenção Básica (SISAB) que deverá integrar os demais sistemas de informação da atenção básica. A operacionalização do SISAB será feita por meio da estratégia do Departamento de Atenção Básica (DAB/SAS/MS) denominada e-SUS Atenção Básica (e-SUS $A B)^{(15)}$. Neste sentido, o estudo apresentado ganha relevância visto a necessidade de que o aprimoramento do SIAB alcance um patamar além do seu aprimoramento tecnológico. É necessário o aperfeiçoamento dos processos que envolvem o SIAB, o fluxo informacional deste sistema, o processo de trabalho da equipe, o modelo assistencial em vigência nas equipes de saúde da família e o reconhecimento das equipes sobre a possibilidade de uso das informações coletadas visando o direcionamento das práticas gerenciais.

A proposta da ESF tem enorme potencial para enfrentar os problemas de saúde na atenção básica, porém esbarra na dificuldade de mudança dos processos de trabalho e de gestão baseados no modelo

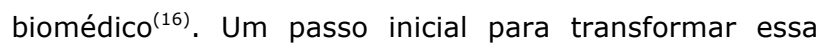


realidade perpassa pela análise das atividades registradas pelos profissionais no SIAB, e se elas condizem com o modelo assistencial que se deseja implantar, um modelo pautado por atividades coletivas com ações e serviços de promoção, prevenção e recuperação da saúde, voltadas para o indivíduo, à família e comunidade ${ }^{(1)}$. Nesta lógica os questionamentos que nortearam este estudo foram: Quais são as atividades registradas no SIAB pelos profissionais das equipes de saúde da família? Quais são predominantes?

Assim, buscou-se analisar o registro das atividades no SIAB, realizadas pelos profissionais da Equipe de Saúde da Família.

\section{MÉTODOS}

Trata-se de estudo descritivo que utilizou registros do SIAB das equipes de saúde da família, implantadas em Minas Gerais, entre os anos de 2001 e 2011. A série histórica compreendeu o último ano com informações disponíveis no Departamento de Informática do Sistema Único de Saúde (DATASUS) no tempo da pesquisa (2011) e os 10 anos anteriores a ele. Sabe-se que nos últimos 10 anos não houve mudanças dos instrumentos de coleta do SIAB, nem de seu fluxo informacional e também de seu manual instrutivo ${ }^{(17)}$. O período de 1998 a 2000 não foi incluído, pois os registros apresentaramse muito baixos ${ }^{(11)}$ em relação ao período de 2001 a 2011 o que pode estar relacionado com a subnotificação do SIAB nos seus primeiros anos de implantação.

De acordo com o Plano Diretor de Regionalização, o estado de MG está dividido em 13 Macrorregiões de Saúde, consideradas a base territorial de planejamento da atenção à saúde, em função das características demográficas, socioeconômicas, geográficas, sanitárias, epidemiológicas, oferta de serviços e relações entre municípios. São elas: Sul, Centro Sul, Centro, Jequitinhonha, Oeste, Leste, Sudeste, Norte, Noroeste, Leste do Sul, Nordeste, Triângulo do Sul, Triângulo do Norte. Essas macrorregiões foram divididas em 76 microrregiões que abrangem o universo de 853 municípios que se diferenciam no nível sociossanitário, porte e forma de gestão e estão distribuídos em 28 Gerências Regionais de Saúde ${ }^{(18)}$.

Os dados foram obtidos junto ao DATASUS, perfazendo 635.788.580 atividades registradas em MG, no período. Ressalta-se que estes registros são provenientes da ficha $D$ do SIAB utilizada por todos os profissionais da equipe mínima de saúde da família (médicos, enfermeiros, técnicos de enfermagem e agentes comunitários de saúde) e denominadas atividades de produção(19). Estes profissionais registram na ficha $D$ as suas atividades realizadas no cotidiano de trabalho da estratégia de saúde da família. As equipes que possuem computador nas unidades de saúde da família consolidam os dados e registram no local, porém as equipes que não são informatizadas consolidam os dados e enviam para as secretarias municipais onde os dados são digitados no sistema. Atualmente, os dados consolidados são enviados automaticamente para o DATASUS que gera os indicadores relacionados à atenção básica ${ }^{(17,19)}$.

Foram analisadas as seguintes variáveis que têm sua definição nas notas técnicas do $\operatorname{SIAB}^{(19)}$ da seguinte forma:

- consultas médicas: registro diário de consultas médicas por procedência e faixa etária;

- atendimentos: registro diário de atendimentos médicos e de enfermagem em residentes no município nas doenças e condições cujo acompanhamento deve se dar de forma sistemática: Puericultura, Pré-natal, Prevenção de Câncer Cérvico-uterino (coleta de Papanicolau), DST/AIDS, Diabetes, Hipertensão Arterial, Hanseníase e Tuberculose);

- solicitações de exames: registro dos encaminhamentos médicos para a realização de exames complementares de qualquer natureza, agrupando-os em: patologia clínica, radiodiagnóstico, citopatológico cérvico-vaginal, ultrassonografia obstétrica e outros. Inclui os casos de encaminhamento para realização de exames complementares na própria unidade de saúde.

- encaminhamentos: neste bloco apenas o médico registra o total de encaminhamentos que fez.

- atendimento especializado: número total de atendimentos específicos para acidente de trabalho.

- visita para inspeção sanitária: número de visitas de inspeção sanitária realizada por profissional de nível superior.

- atendimento individual: número de atendimentos individuais por profissional de nível superior (de enfermeiro e outros profissionais de nível superior, exceto médicos e odontólogos).

- procedimentos individuais: procedimentos realizados por profissional de nível médio (injeções, curativos, retirada de pontos e terapia de reidratação oral). 
- atendimento em grupo: atividade de educação em saúde, com no mínimo 10 pessoas e duração mínima de 30 minutos realizada por profissional de nível superior e médio, exceto agente comunitário de saúde;

- procedimentos coletivos: atividades de promoção e prevenção, de baixa complexidade, em saúde bucal;

- reuniões: realizadas pelos ACS com 10 ou mais pessoas e duração mínima de 30 minutos. Tem o objetivo de disseminar informações, discutir estratégias de superação de problemas de saúde ou de contribuir para a organização comunitária;

- visitas domiciliares: visitas domiciliares realizadas qualquer que seja a finalidade, exceto as de Inspeção Sanitária. As visitas domiciliares podem ser realizadas por profissional de nível superior, profissional de nível médio e por agente comunitário de saúde.

Os dados foram obtidos apenas por um pesquisador devidamente treinado. Estes dados foram isolados e não houve associação de variáveis. Realizou-se análise descritiva das informações do estado e de suas macrorregiões, segundo o tipo de atividade e a categoria profissional de saúde da família, utilizando-se o software estatístico Statístical Pocckage for the Social Sciences (SPSS $®$ ) 17.0 .

As informações utilizadas neste estudo foram acessadas em banco de dados oficiais e de acesso livre.

\section{RESULTADOS}

Entre as 635.788 .580 atividades de produção registradas no SIAB em MG, verificou-se o predomínio de atividades de cunho individual (aquelas focadas na assistência a apenas um indivíduo), destacando-se as consultas médicas $(25,1 \%)$, os atendimentos individuais $(16,9 \%)$, os atendimentos $(15,0 \%)$, os procedimentos individuais $(11,9 \%)$ e a solicitação de exames $(10,6 \%)$, que somadas, totalizaram $79,5 \%$ dos registros. As atividades de caráter coletivo (focadas na assistência a um grupo de indivíduos) foram as menos registradas, variando de $0,3 \%$ (reuniões de ACS com a população) a $9,5 \%$ (procedimentos coletivos / odontologia). As visitas domiciliares e de inspeção sanitária representaram 7,6\% e $0,4 \%$ dos registros, respectivamente. O registro das atividades coletivas totalizaram 10,2\% (Tabela 1) retratando grande divergência em relação à totalidade das atividades de caráter individual registradas $(81,5 \%)$.

Tabela 1: Atividades registradas no Sistema de Informação da Atenção Básica em Minas Gerais entre 2001 e 2011. Minas Gerais, Brasil, 2012.

\begin{tabular}{|c|c|c|}
\hline Atividades & n (milhões) & $\%$ \\
\hline Consultas médicas & 159.396 & 25,1 \\
\hline Atendimentos individuais & 107.407 & 16,9 \\
\hline Atendimentos & 95.203 & 15,0 \\
\hline Procedimentos individuais & 75.579 & 11,9 \\
\hline Solicitação de exames & 67.627 & 10,6 \\
\hline Procedimentos coletivos (Odontologia) & 60.163 & 9,5 \\
\hline Visitas domiciliares & 48.170 & 7,6 \\
\hline Encaminhamentos & 11.201 & 1,8 \\
\hline Atendimentos em grupo & 5.268 & 0,8 \\
\hline Visitas para inspeção sanitária & 2.468 & 0,4 \\
\hline Reuniões & 2.186 & 0,3 \\
\hline Atendimento especializado & 1.115 & 0,2 \\
\hline TOTAL & 635.788 & 100 \\
\hline
\end{tabular}

Fonte: DATASUS, 2012.

Na análise da distribuição dos registros de saúde, a média de atividades coletivas (atendimentos em grupos e reuniões realizadas por ACS) e de visitas domiciliares foram $1,1 \%$ e $7,6 \%$ respectivamente em relação ao número de atividades registradas no SIAB (Tabela 2 ).

Tabela 2: Distribuição média de atividades coletivas e visitas domiciliares comparadas ao total de registros de atividades no Sistema de Informação da Atenção Básica em Minas Gerais, entre 2001 e 2011. Minas Gerais, Brasil, 2012.

\begin{tabular}{ccc}
\hline Atividades & Média (\%) (mínimo - máximo) & Dp \\
\hline Atividades coletivas & $1,1(0,6-2,2)$ & 0,40 \\
Visitas domiciliares & $7,6(4,8-11,6)$ & 4,4 \\
\hline Fonte: DATASUS, 2012.
\end{tabular}


Ao analisar estes dados segundo as macrorregiões de saúde verificou-se uma distribuição homogênea dos percentuais de atividades coletivas, entretanto, em relação às visitas domiciliares verificou-se uma variação de $1,6 \%$ a $11,6 \%$. As regiões com maiores índices foram Sul, Triângulo do Sul e Centro Sul (Tabela 3).

Tabela 3: Percentual de atividades coletivas e visitas domiciliares comparadas

ao total de registros de atividades no Sistema de Informação da Atenção Básica,

segundo as macrorregiões de saúde de Minas Gerais, entre 2001 e 2011. Minas Gerais, Brasil, 2012.

\begin{tabular}{ccc}
\hline Macrorregiões de Saúde de Minas Gerais & Atividades Coletivas (\%) & Visitas domiciliares (\%) \\
\hline Centro & 1,3 & 4,8 \\
Sul & 0,8 & 11,6 \\
Norte & 2,2 & 5,8 \\
Sudeste & 1,0 & 1,6 \\
Leste & 1,0 & 7,2 \\
Triângulo do Norte & 1,0 & 8,2 \\
Oeste & 0,9 & 5,8 \\
Nordeste & 0,6 & 10,8 \\
Centro Sul & 1,1 & 11,0 \\
Triângulo do Sul & 1,1 & 6,5 \\
Leste do Sul & 0,6 & 5,3 \\
Noroeste & 0,6 & 6,4 \\
\hline Jequitinhonha & 0,8 & $\mathbf{7 , 9}(\mathbf{4 , 8 - 1 1 , 6 )}$
\end{tabular}

Fonte: DATASUS, 2012

Dentre as categorias profissionais que mais realizaram registros das atividades, destacam-se os médicos e os enfermeiros que, juntos, são responsáveis por mais da metade dos registros no SIAB $(67,6 \%)$, como mostra a Tabela 4.

Tabela 4: Atividades registradas no Sistema de Informação da Atenção Básica segundo a categoria profissional, em Minas Gerais, entre 2001 e 2011. Minas Gerais, Brasil, 2012.

\begin{tabular}{ccc}
\hline Atividades & n (em milhões) & \% \\
\hline Médico & 246.846 & 38,8 \\
Profissional de nível médio & 100.970 & 15,9 \\
Médico e enfermeiro & 95.203 & 15,0 \\
Enfermeiro & 88.019 & 13,8 \\
Profissional da odontologia & 60.163 & 9,5 \\
Outro profissional de nível superior & 34.639 & 5,4 \\
Profissional de nível superior e médio & 5.268 & 0,8 \\
Agente comunitário de saúde & 2.186 & 0,3 \\
Diversos* & 2.468 & 0,4 \\
\hline Total & $\mathbf{6 3 5 . 7 6 6}$ & $\mathbf{1 0 0}$ \\
\hline
\end{tabular}

*Trata-se de inspeção sanitária realizada por profissionais de nível superior. Fonte: DATASUS, 2012.

\section{DISCUSSÃO}

As atividades predominantes registradas no SIAB em MG são de caráter individual focadas no fluxo da consulta individual, solicitação de exames e encaminhamentos, retratando, assim, o registro de informações de um modelo centrado na doença e no profissional de saúde, onde predominam as consultas e procedimentos, em detrimento de atividades coletivas focadas na assistência em grupo, para a comunidade e a família. Não há na literatura ou nos protocolos do Ministério da Saúde uma parametrização ideal para o número de atividades individuais realizadas, bem como para atividades em grupo (de foco coletivo). Entretanto, há a proposta de inversão do modelo tradicional da atenção básica que a
ESF deseja implantar, mediante a priorização de ações de promoção e proteção da saúde das famílias e comunidades $^{(1)}$ dando ênfase às ações de promoção e prevenção da saúde na perspectiva da integralidade da atenção(20).

Apesar de o SIAB ter sido criado para gerenciar as informações geradas pela ESF, este sistema não está adequado às demandas informacionais do processo de trabalho que se estabelece no cotidiano das equipes. Há a necessidade de adaptar este sistema a atenção básica com vistas a ser possível o registro de informações advindas das atividades coletivas desenvolvidas pelas equipes $^{(7-8)}$. Destaca-se que o SIAB não possui, principalmente na ficha $D$, campos onde possam 
registrar de forma real as atividades coletivas desenvolvidas $^{(6)}$. Pela óptica de enfermeiros e ACS os instrumentos de coleta do SIAB não permitem o registro de várias atividades que são realizadas no cotidiano de trabalho das equipes, tais como os grupos operativos, atividades com o conselho local, orientações, acolhimento, dentre outras ${ }^{(14)}$. Além disso, outros estudos destacam que os profissionais avaliam o SIAB como um sistema que não é capaz de captar a realidade de trabalho que se estabelece na atenção primária, a começar por suas fichas que são desatualizadas, parte da necessidade do Estado por informações e não considera a realidade local nos diversos municípios brasileiros ${ }^{(10-12)}$. Isto poderia estar limitando o registro de outras atividades, que de fato acontecem nas equipes de saúde da família.

A falta de capacitação para uso do SIAB é outro problema crônico apresentado no decorrer do tempo. Vários estudos apontam que não há uma capacitação sistemática para os profissionais visando à compreensão de seus instrumentos, seu fluxo, a análise de seus dados e o planejamento das ações a partir deste sistema ${ }^{(10,12,14)}$. Desta feita, se os profissionais não são capacitados adequadamente e não compreendem a totalidade das variáveis presentes em seus instrumentos é possível que a qualidade destes dados não esteja adequada. Isto pode influenciar na geração de indicadores fidedignos e que norteie de forma assertiva a gestão da atenção básica.

É necessário considerar que existem dificuldades para mudança do foco curativo na prática dos profissionais de saúde, em função do modelo hegemônico de formação desses profissionais dominante em nosso país ${ }^{(4)}$. A concepção do SIAB, a partir de seus instrumentos de coleta priorizando o registro de atividades individuais reforça esta prática profissional centrada na doença. Assim, a prática do profissional e a concepção dos sistemas de informação necessitam deslocar da área curativa e individualizada para a produção de serviços em unidades básicas de saúde com ênfase nas ações de promoção e prevenção de saúde em bases coletivas.

Estudo realizado com médicos de saúde da família identificou que $65 \%$ dos entrevistados acham importante a realização de atividades coletivas, entretanto, por algum motivo, não destinam tempo adequado a essa atividade e ainda relataram que a divisão de tempo entre consultas individuais e atividades coletivas não são condizentes com as necessidades da área de abrangência da $\mathrm{ESF}^{(21)}$.
As atividades em grupo possuem importante papel na ESF, uma vez que têm o propósito de capacitar o indivíduo para o autocuidado e são realizadas em grupo onde ocorre troca de conhecimento e experiências, sobre determinado assunto, entre paciente-profissional e paciente-paciente ${ }^{(22)}$.

Por outro lado, os próprios usuários, influenciados pelo modelo biomédico, ao qual foram submetidos há vários anos, não se empoderam de novas práticas em saúde, especialmente as ações coletivas, pois em seu imaginário, o cuidado e a cura dependem do uso de procedimentos e de produtos médicos fornecidos pelos profissionais que atuam como mediadores ${ }^{(4)}$.

A atenção básica exige conhecimentos e práticas muito complexas pelas interferências da subjetividade e necessária negociação da terapêutica. Isso leva os profissionais a negar essa complexidade e a desprezar a atenção básica priorizando assim, o atendimento clássico embasado em práticas hospitalares ${ }^{(23)}$.

Outro ponto importante destacado nos atributos da Atenção Primária em Saúde (APS) é o vínculo entre a população e a fonte de serviços, cujo produto reflete na acessibilidade, na utilização dos serviços de saúde por meio do acolhimento humanizado e consequentemente, na satisfação do usuário ${ }^{(24)}$.

As visitas domiciliares têm importante contribuição na construção do vínculo, além de constituir-se de ações com foco educativo que busca acompanhar o indivíduo e a família de forma integral e contínua, orientar o autocuidado, a manutenção e a promoção da saúde ${ }^{(25)}$. Contribui também para conhecer a situação de saúde, identificar suas necessidades, bem como, realizar a busca ativa da população.

Os resultados desta pesquisa apontam uma distribuição heterogênea na quantidade registrada de visitas domiciliares entre as regiões do Estado. Pressupõe-se que esse resultado relaciona-se de um lado com as características distintas entre as regiões, como a distribuição populacional, densidade demográfica, as características socioeconômicas, e de outro, com a organização da assistência em saúde local.

Com relação aos profissionais, os que mais realizaram o registro das atividades são os médicos e enfermeiros que juntos são responsáveis pela maior parte dos registros de atividades individuais. Esse resultado já era esperado, uma vez que, o médico e o enfermeiro são os profissionais com maior demanda de atividades na ESF. Entretanto é importante envolver as demais categorias profissionais, visto a importância do 
olhar multiprofissional e da troca de informações entre a equipe.

\section{CONCLUSÃO}

As principais atividades registradas pelo conjunto das equipes de saúde da família de MG são as individuais, como consultas e atendimentos, retratando a concepção do próprio sistema que prioriza na coleta as atividades individuais e focadas em procedimentos, bem como reforça na disseminação das informações esta mesma lógica.

Esse estudo reforça a necessidade de adaptar o SIAB de acordo com a sua proposta de implantação, ou seja, organizar e aprimorar a qualidade das informações para subsidiar o monitoramento, a avaliação e o planejamento, segundo as prioridades de saúde da população. É preciso buscar o aprimoramento do SIAB além dos aspectos tecnológicos enfatizando o seu fluxo informacional, os processos de trabalho que instrumentaliza, a realidade diversificada das regiões

\section{REFERÊNCIAS}

1. Espíndola PS, Lemos CSL, Reis LBM. Perfil do profissional de nível superior na Estratégia Saúde da Família. Rev. bras. promoç. Saúde [Internet]. 2011 [acesso em: 31 dez 2014];24(4):367-75. Disponível em:

http://www.unifor.br/images/pdfs/rbps/artigo10_2011.4.pdf. 2. Costa GD, Cotta RMM, Ferreira MLSM, Reis JR, Franceschini SCC. Saúde da família: desafios no processo de reorientação do modelo assistencial. Rev Bras Enferm [Internet]. 2009 [acesso em: 31 dez 2014];62(1):113-8. Disponível em: http://dx.doi.org/10.1590/S0034-71672009000100017. 3. Horta NC, Sena RR, Silva MEO, Oliveira SR, Rezende VA. A prática das equipes de saúde da família: desafios para a promoção de saúde. Rev Bras Enferm [Internet]. 2009 [acesso em: 31 dez 2014];62(4):524-9. Disponível em: http://dx.doi.org/10.1590/S0034-71672009000400005. 4. Junges JR, Selli L, Soares NA, Fernandes RBP, Schereck M. Processos de trabalho no Programa Saúde da Família: atravessamentos e transversalidades. Rev Esc Enferm USP [Internet]. 2009 [acesso em: $31 \mathrm{dez} 2014$ ];43(4):937-44. Disponível em: http://dx.doi.org/10.1590/S008062342009000400028.

5. Ministério da Saúde. Sistema de informação da assistência básica (SIAB): indicadores 2006 [Internet]. Brasília: Ministério da Saúde; 2009 [acesso em: 31 dez 2014]. Disponível em: http://bvsms.saude.gov.br/bvs/publicacoes/sistema_informacao _atencao_basica_siab2006_p1.pdf.

6. Thaines GHLS, Bellato R, Faria APS, Araújo LFS. Produção, fluxo e análise de dados do sistema de informação em saúde: um caso exemplar. Texto Contexto Enferm [Internet]. 2009 [acesso em: 31 dez 2014];18(3):466-74. Disponível em: http://dx.doi.org/10.1590/S0104-07072009000300009.

7. Radigonda B, Conchon MF, Carvalho WO, Nunes EFPA. Sistema de informação da atenção básica e sua utilização pela equipe de saúde da família: uma revisão integrativa. Espaç. saúde (Online). [Internet]. 2010 [acesso em: $31 \mathrm{dez} 2014$ ];12(1):38-47.

Disponível em:

http://www.uel.br/revistas/uel/index.php/espacoparasaude/articl e/view/9236. brasileiras e as demandas informacionais da equipe multidisciplinar.

É necessário aprimorar os instrumentos de coleta para que a consolidação dos dados represente a diversidade de atividades realizadas no cotidiano das equipes de saúde da família. Endossamos o fato de que o SIAB, da forma como está estruturado, está reforçando o registro de atividades de caráter individual, tanto em sua coleta, como na disseminação de seus consolidados por meio do site do DATASUS.

Entretanto, não podemos afirmar que de fato as equipes de saúde da família estejam realizando apenas atividades de caráter individual, esta é uma limitação deste estudo. É preciso continuar investigando a realidade das atividades desenvolvidas nas equipes de saúde da família, o seu predomínio em detrimento de outras atividades e os motivos que estão desencadeando este fenômeno. Outro questionamento que emerge é se a proposição do SISAB, com vistas ao aperfeiçoamento do SIAB, é suficiente para atender as demandas de informações das equipes de saúde da família.

8. Cavalcante RB, Pinheiro MMK, Bernardes MFVG, Cunha SGS, Santos CS. Fluxo informacional do Sistema de Informação da Atenção Básica. R. Enferm. Cent. O. Min [Internet]. 2011 [acesso em: 31 dez 2014];1(4):523-36. Disponível em: http://www.seer.ufsj.edu.br/index.php/recom/article/view/161. 9. Duarte MLC, Tedesco JR, Parcianello RR. The use of information systems in family health strategies: nurses'perceptions. Rev Gaucha Enferm [Internet]. 2012 [acesso em: 31 dez 2014];33(4):111-7. Disponível em: http://dx.doi.org/10.1590/S1983-14472012000400014. 10. Marcolino JS, Scochi MJ. Informações em saúde: o uso do SIAB pelos profissionais das Equipes de Saúde da Família. Rev Gaucha Enferm [Internet]. 2010 [acesso em: $31 \mathrm{dez}$ 2014];31(2):314-20. Disponível em: http://dx.doi.org/10.1590/S1983-14472010000200016. 11. Cavalcante RB, Bernardes MFVG, Gontijo TL, Guimarães EAA, Oliveira VC. Sistema de informação da atenção básica: potencialidades e subutilização no processo decisório. Cogitare enferm. [Internet]. 2013 [acesso em: $31 \mathrm{dez} 2014$ ];18(3):4607. Disponível em: http://dx.doi.org/10.5380/ce.v18i3.33555. 12. Silva AS, Laprega MR. Avaliação crítica do Sistema de Informação da Atenção Básica (SIAB) e de sua implantação na região de Ribeirão Preto, São Paulo, Brasil. Cad Saude Publica [Internet]. 2005 [acesso em: $31 \mathrm{dez} 2014$ ];21(6):1821-8. Disponível em: http://dx.doi.org/10.1590/S0102$311 \times 2005000600031$.

13. Cavalcante RB, Pinheiro MMK, Guimarães EAA. Sistema de informação da atenção básica como instrumento de poder. Rev enferm UFPE on line [Internet]. 2013 [acesso em: $31 \mathrm{dez}$ 2014];7(2):371-80. Disponível em:

http://www.revista.ufpe.br/revistaenfermagem/index.php/revista /article/view/3795.

14. Lima AP, Corrêa ACP, Oliveira QC. Conhecimento de Agentes Comunitários de Saúde sobre os instrumentos de coleta de dados do SIAB. Rev Bras Enferm [Internet]. 2012 [acesso em: $31 \mathrm{dez}$ 2014];65(1):121-7. Disponível em:

http://dx.doi.org/10.1590/S0034-71672012000100018. 15. Portaria no 1412, de 10 de julho de 2013 (BR). Institui o Sistema de Informação em Saúde para a Atenção Básica (SISAB). Diário Oficial da União. 11 jul. 2013 [acesso em: 31 dez 
2014]. Disponível em:

http://bvsms.saude.gov.br/bvs/saudelegis/gm/2013/prt1412_10 _07_2013.html.

16. Franco TB, Merhy EE. Programa de Saúde da Família (PSF): contradições de um programa destinado à mudança do modelo tecnoassistencial. In: Merhy EE, Magalhães HM, Rimoli J, Franco TB, Bueno WS. O trabalho em saúde: olhando e experienciando o SUS no cotidiano. São Paulo: Hucitec; 2003. p. 55-124.

17. Ministério da Saúde. SIAB: manual do Sistema de Informação da Atenção Básica [Internet]. Brasília: Ministério da Saúde; 2003 [acesso em: $31 \mathrm{dez} 2014$ ]. Disponível em: http://www.psfmonteazul.org.br/manuais/siab.pdf. 18. Secretaria de Estado de Saúde de Minas Gerais. Análise de situação de saúde - Minas Gerais 2010 [Internet]. Belo Horizonte: Secretaria de Estado de Saúde de Minas Gerais; 2010 [acesso em: $31 \mathrm{dez} 2014]$. Disponível em:

http://www.saude.mg.gov.br/images/documentos/publicacao_su bsec_saude_FINAL.pdf.

19. DATASUS [Internet]. Brasilia: Ministério da Saúde (BR) [acesso em: 31 dez 2014] Sistema de Informação da Atenção Básica: Situação de Saúde - Notas Técnicas. Disponível em: http://tabnet.datasus.gov.br/cgi/siab/siabsdescr.htm.

20. Nascimento MS, Nascimento MAA. Prática da enfermeira no Programa de Saúde da Família: a interface da vigilância da saúde versus as ações programáticas em saúde. Cien Saude Colet [Internet]. 2005 [acesso em: $31 \mathrm{dez} 2014$ ];10(2):333-45. Disponível em: http://dx.doi.org/10.1590/S141381232005000200011.

21. Vasconcelos FGA, Zaniboni MRG. As dificuldades do trabalho médico no PSF. Cien Saude Colet [Internet]. 2011 [acesso em:

31 dez 2014];16(Supl. 1):1494-504. Disponível em: http://dx.doi.org/10.1590/S1413-81232011000700085. 22. Gandra FPP, Silva KC, Castro CF, Esteves EA, Nobre LN. Efeito de um programa de educação no nível de conhecimento e nas atitudes sobre Diabetes Mellitus. Rev. bras. promoç. Saúde [Internet]. 2011 [acesso em: $31 \mathrm{dez} 2014$ ];24(4):322-31. Disponível em:

http://www.unifor.br/images/pdfs/rbps/artigo5_2011.4.pdf. 23. Cunha GT. A construção da clínica ampliada na atenção básica. São Paulo: Hucitec; 2005. 212 p.

24. Starfield B. Atenção Primária: equilíbrio entre necessidades de saúde, serviços e tecnologia. Brasília: Ministério da Saúde/ UNESCO; 2002. 726 p.

25. Lima AN, Silva L, Bousso RS. A visita domiciliária realizada pelo agente comunitário de saúde sob a ótica de adultos e idosos. Saude soc. [Internet]. 2010 [acesso em: $31 \mathrm{dez}$ 2014];19(4):889-97. Disponível em:

http://dx.doi.org/10.1590/S0104-12902010000400015.

Artigo recebido em 15/02/2013.

Aprovado para publicação em 30/05/2014.

Artigo publicado em 31/12/2014. 